\section{Qualidade de vida e percepção da doença entre portadores de hipertensão arterial}

\author{
Quality of life and perception of illness among \\ individuals with high blood pressure
}

\author{
${ }^{1}$ Hospital São José de \\ Doenças Infecciosas, \\ Fortaleza, Brasil. \\ 2 Curso de Pós-graduação em \\ Enfermagem, Universidade \\ Federal do Ceará, Fortaleza, \\ Brasil. \\ ${ }_{3}$ Curso de Pós-graduação em \\ Cuidados Clínicos em Saúde, \\ Universidade Estadual do \\ Ceará, Fortaleza, Brasil. \\ Correspondência \\ D. M. S. Brito \\ Hospital São José de Doenças \\ Infecciosas. \\ Rua Nestor Barbosa 315 \\ Fortaleza, $C E$ \\ 60455-610, Brasil. \\ danemel6@hotmail.com
}

\begin{abstract}
This study aimed to investigate quality of life in individuals with arterial hypertension and to associate the results with the patients' perceived severity of illness. The sample included 113 patients with arterial hypertension followed up in a clinic in Fortaleza, Ceará State, Brazil, from May to August 2002, using an international scale for assessing quality of life, the SF-36. Of the entire sample of patients, $77 \%$ were women, a large proportion were over 50 years of age, living with family, and with some kind of occupation. Assessment with the SF-36 showed compromised quality of life in different domains, although patients viewed their illness as not serious, and curable. These circumstances can interfere in follow-up of their illness over time, which can in turn jeopardize their quality of life. In conclusion, use of the SF-36 to assess individuals with arterial hypertension produced significant results, indicating generally compromised quality of life. According to these results, hypertension jeopardizes the patients' social and physical dimensions, but this situation can be changed by the provision of qualified services.
\end{abstract}

Questionnaires; Quality of Life; Hypertension
Daniele Mary Silva de Brito 1

Thelma Leite de Araújo 2

Marli Teresinha Gimeniz Galvão 2

Thereza Maria Magalhães Moreira ${ }^{3}$

Marcos Venícios de Oliveira Lopes 2

\section{Introdução}

A hipertensão arterial é considerada um dos fatores de risco mais importantes para o desenvolvimento de doenças cardiovasculares e sofre influência multifatorial, com destaque para o estilo de vida 1.

Conforme se indica, concomitante à terapêutica para o controle da hipertensão arterial, os indivíduos devem adotar um estilo de vida saudável, eliminando hábitos que constituam fatores de risco para a doença. Entretanto, no acompanhamento à saúde dos pacientes, segundo se observa, é grande a dificuldade para a aquisição de hábitos saudáveis, pois a tomada de decisão com vistas à superação de hábitos nocivos à saúde, apesar de necessária, constitui uma decisão pessoal.

Diante dessas polêmicas, é cada vez mais freqüente o interesse pelo assunto e a produção de pesquisas sobre avaliações de qualidade. A expressão qualidade de vida pode indicar diferentes aspectos do cotidiano do indivíduo ou de grupos específicos.

De acordo com a definição da Organização Mundial da Saúde (OMS), qualidade de vida é a percepção do indivíduo sobre sua posição na vida, considerada no contexto da cultura e dos valores nos quais vive e elabora seus objetivos, expectativas, padrões e preocupações 2,3 . Dessa forma, a percepção que o paciente possui da doença influenciará na sua qualidade de vida, 
interferindo em suas condições de saúde e em outros aspectos gerais da vida pessoal 4 .

As experiências clínicas e as mudanças comportamentais dos indivíduos em face de tratamentos específicos propiciaram a instituição de medidas na avaliação de respostas do perfil de saúde dos pacientes. Concomitantes a esse fato surgiram propostas de avaliação da qualidade de vida obtidas por instrumentos ou escalas.

Um desses instrumentos é o SF-36 (Medical Outcomes Study 36-Item Short-Form Health Survey) formulário genérico que teve sua utilidade demonstrada na literatura internacional 5 . No Brasil, foi validado e objeto de adaptação cultural 6, sendo utilizado em diferentes áreas da saúde. Sua finalidade maior é analisar a compreensão integral do indivíduo sobre sua saúde, no intuito de ajudá-lo a tomar decisões sobre seu tratamento 5 .

Este estudo teve como objetivos investigar a qualidade de vida dos portadores de hipertensão arterial, utilizando o SF-36, e associar estes resultados à percepção dos pacientes sobre a gravidade da hipertensão.

\section{Metodologia}

Desenvolveu-se um estudo observacional transversal, que buscou um retrato instantâneo da qualidade de vida e da percepção da gravidade da hipertensão arterial entre 113 portadores deste fator de risco de ambos os sexos.

O estudo foi conduzido em uma Unidade Básica de Saúde da Família (UBASF), constituída por seis equipes com ações de ordens primária e secundária em programas destinados à saúde ao longo do ciclo vital. Envolveu os portadores de doenças crônico-degenerativas do Município de Fortaleza, Estado do Ceará, Brasil, no período de maio a agosto de 2002.

Para seleção, adotaram-se como critérios de inclusão: serem pacientes de ambos os sexos; com idade igual ou superior a 18 anos; apresentarem diagnóstico médico de hipertensão arterial primária há mais de um ano; estarem cadastrados e acompanhados no programa de hipertensão da unidade; e estarem conscientes, orientados e capazes de se comunicarem oralmente. Não houve recusa dos pacientes em participar do estudo, portanto, foram avaliados todos os inscritos na UBASF.

Conduziu-se, então, entrevista individual realizada pela investigadora principal, no domicílio do paciente, com tempo de, aproximadamente, trinta minutos.

Para coleta de dados utilizou-se um instrumento com roteiro que permitia a caracterização dos principais dados sócio-demográficos (sexo, idade, renda familiar, instrução, situação funcional) dos pacientes e a aplicação do SF-36.

O SF-36 é um formulário genérico de avaliação de qualidade de vida para indivíduos acima de 14 anos, auto-aplicável ou submetido em entrevista face a face ou por telefone. Foi traduzido e adaptado em 29 países e utilizado entre diferentes grupos de pacientes, incluindo portadores de hipertensão arterial. Apresenta 36 itens ou questões e é dividido em nove domínios, a saber: 1. Estado geral de saúde (GS), com dez itens; 2. Evolução do estado de saúde (EV), que realiza comparação das condições de saúde atual às percebidas há um ano, com cinco itens;

3. Capacidade funcional (CF), com dez itens;

4. Desempenho físico (DF), com dez itens;

5. Aspectos emocionais (AE), com dez itens;

6. Aspectos sociais (AS), com dez itens;

7. Dor (DR), com dez itens;

8. Vitalidade (VT), com dez itens;

9. Saúde mental (SM), com cinco itens.

Para cada item, o paciente deveria escolher apenas uma opção.

Para cada domínio do SF-36, os itens são codificados e transformados em escala de zero a 100 pontos, utilizando-se pontuação e interpretação própria da escala 5 . Dessa forma, obtém-se um escore médio: o maior escore indica melhor estado de saúde ou qualidade de vida, e os escores menores pior situação ou qualidade de vida prejudicada.

Os dados foram armazenados e analisados no programa SPSS 10.0 (SPSS Inc., Chicago, Estados Unidos). Para descrever os valores dos domínios do SF-36 foram empregados média e desvio padrão (média \pm DP) e aplicado o teste de Mann-Whitney nas análises de distribuição dos valores obtidos pelos 113 portadores de hipertensão arterial nos diferentes domínios do SF-36; das medidas descritivas dos domínios do SF-36 e percepção da gravidade da doença; e das medidas descritivas dos domínios do SF-36 e percepção da cura da doença referida pelos avaliados. $\mathrm{O}$ nível de significância adotado foi de 0,05.

Para o estudo da comparação entre a percepção da gravidade e cura da doença foram analisados oito domínios. Excluiu-se o domínio "evolução do estado de saúde" em virtude de seu resultado ser apresentado em freqüência simples e porcentagem.

Para avaliar a percepção da gravidade da doença, do tratamento e o tempo de tratamento da hipertensão, utilizou-se uma questão proposta por pesquisadores 7,8, a qual oferece três opções diferentes de resposta: (i) curável com tratamento; (ii) a doença pode ser curada, mesmo sem tratamento; e (iii) não cura a doença, mas con- 
trola. Após a coleta de dados, as respostas foram somadas em duas opções, curável e não curável, em decorrência do segundo item ter sido apontado apenas uma vez. Dessa forma, por orientação estatística, esse item foi aglutinado àqueles que responderam que a doença era curável.

O estudo seguiu as recomendações da Resolução no. 196/96, do Conselho Nacional de Saúde de 1996. Inicialmente o protocolo foi apreciado e autorizado pelo Comitê de Ética em Pesquisa da Universidade Federal do Ceará e, para todos os pacientes foram oferecidas informações sobre a pesquisa e, após anuência, assinaram o Termo de Consentimento Livre e Esclarecido.

\section{Análise e discussão dos resultados}

Dos 113 portadores de hipertensão arterial, a maioria era do sexo feminino $(\mathrm{n}=87 ; 77 \%)$. O maior número de mulheres atendidas nos programas de hipertensão arterial é observado talvez em razão das características da mulher como cuidadora e mais atenta aos aspectos de sua saúde.

As idades variaram de 25 a 65 anos, mas $69 \%$ do total se encontravam em idade acima de 50 anos. Na medida em que são acrescidos anos de vida à população a pressão arterial se eleva e, em conjunto com outras moléstias cardiovasculares, torna-se fator de grande repercussão nos índices de morbidade e mortalidade geriátrica. Atualmente, a ocorrência de hipertensão arterial em idosos brasileiros alcança o percentual de $65 \%$, com a maioria apresentando elevação isolada ou predominante da pressão sistólica, que mostra forte relação com eventos cardiovasculares 9 .
Do total, nenhum dos entrevistados havia concluído ensino superior e 48 (42\%) informaram nenhuma escolaridade [analfabetos]. Quando indagados sobre a situação conjugal, 63 (55,8\%) referiram relacionamento estável, 27 $(23,9 \%)$ eram viúvos e os demais encontravam-se em outras situações. Em relação à renda familiar, variava de um a três salários mínimos (o valor do salário mínimo da época era de $\mathrm{R} \$ 240,00$ ) e observou-se entre os entrevistados 21 (18,6\%) aposentados.

Já se mencionou que o conjunto dos pacientes estudados era majoritariamente do sexo feminino, com acesso escolar limitado e idade avançada, contando com escassa renda financeira, advinda mensalmente dos proventos das aposentadorias.

Na análise dos diferentes domínios da escala de qualidade de vida, o maior comprometimento ocorreu no "estado geral de saúde" e "dor", considerando-se as médias inferiores a 60 pontos. Os demais domínios, entretanto, apresentam valores ou qualidade de vida comprometida entre 60,8 e 69,36, índices também considerados baixos (Tabela 1). Dessa forma, verifica-se o comprometimento em todos os domínios da escala SF-36, já que a melhor qualidade de vida deveria ser 100 pontos, sugerindo que a hipertensão, por sua cronicidade, pode levar ao comprometimento da qualidade de vida do indivíduo.

Ao se analisar o domínio "estado geral de saúde", encontrou-se o menor índice (média = $53,58)$, provavelmente em decorrência das manifestações clínicas relacionadas à etiologia da hipertensão arterial e ao tratamento instituído.

Ao se observar os valores do domínio "capacidade funcional”, que investigou a presença e

Tabela 1

Valores obtidos nos diferentes domínios do SF-36, entre os 113 portadores de hipertensão arterial. Fortaleza, Ceará, Brasil, 2003.

\begin{tabular}{lcccc}
\hline Domínios * & Itens & Média \pm DP & Variação & $\mathbf{p} * *$ \\
\hline Estado geral de saúde & 5 & $53,58 \pm 22,02$ & $0-95$ & 0,017 \\
Capacidade funcional & 10 & $66,28 \pm 27,88$ & $0-100$ & 0,184 \\
Desempenho físico & 4 & $60,8 \pm 39,9$ & $0-100$ & 0,768 \\
Aspectos emocionais & 3 & $64,6 \pm 40,65$ & $0-100$ & 0,183 \\
Aspectos sociais & 2 & $69,36 \pm 30,62$ & $0-100$ & 0,96 \\
Dor & 2 & $56,74 \pm 32,61$ & $0-100$ & 0,211 \\
Vitalidade & 4 & $63,32 \pm 23,68$ & $0-100$ & 0,818 \\
Saúde mental & 5 & $65,45 \pm 22,06$ & $4-100$ & 0,853 \\
\hline
\end{tabular}

* Excluiu-se desta tabela o domínio "evolução do estado de saúde" porque se realiza comparação das condições de saúde atual com as percebidas há um ano, não sendo empregadas média ou desvio padrão;

** Teste Mann-Whitney. 
extensão das limitações relacionadas à capacidade e atividade física, encontrou-se o total de 66,28 pontos, sugerindo prejuízo na capacidade funcional dos portadores de hipertensão.

Ao se avaliar o domínio "desempenho físico", que pesquisava limitações no tipo e quantidade de trabalho e o quanto estas limitações dificultam sua realização e das atividades de vida diária individuais, este fato demonstra que, apesar da idade da maioria dos portadores de hipertensão e do tempo da doença no organismo, os pacientes não se mostraram incapazes fisicamente e conseguiam desenvolver algumas atividades cotidianas. Encontrou-se, entretanto, prejuízo da qualidade de vida (média $=60,8$ ).

Pelos índices apontados entre os domínios "capacidade funcional" e "desempenho físico", evidencia-se que a noção geral de doença apresentada pelos entrevistados está relacionada com a impossibilidade de andar e de trabalhar. Portanto, para os entrevistados, estar doente é ficar impossibilitado de exercer suas atividades diárias. Essa forma de perceber a doença, associada à incapacidade física para o trabalho, pode ser uma das dificuldades para o seguimento do tratamento anti-hipertensivo, uma vez que a hipertensão é considerada uma doença silenciosa, que não apresenta sinais visíveis e que, portanto, não seria associada pela população à incapacidade física para o trabalho. Pode, então, ocorrer diminuição do limite entre o "estar doente" e o "estar com saúde" pelo fato de os indivíduos manterem suas posições cotidianas até surgirem as complicações e, conseqüentemente, as limitações físicas, psicológicas ou sociais delas decorrentes, fato que pode vir a comprometer a qualidade de vida dos portadores 10 .

O domínio "aspectos emocionais" investiga a participação do paciente em atividades de trabalho e o tempo dispensado ao autocuidado. O índice médio de 64,6 pontos indica prejuízos na vida dos pacientes. Esse aspecto pode ser entendido como um estado de saúde/doença intermediário, pois são condições que causam sensação desagradável nas pessoas, impossibilitando-as de se considerarem saudáveis. Outro aspecto é a freqüência entre médicos e pacientes de rotularem a doença hipertensiva como "emocional" e "nervosa", evidenciando uma explicação reducionista da doença. Dessa forma, os pacientes parecem se isentar da responsabilidade de buscar o controle da pressão, mantendo a crença de que o seu estado emocional depende dos outros. Isso, muito provavelmente, implicará o comprometimento da qualidade de vida 11.

Ao avaliar os "aspectos sociais", seus itens demonstram a integração da pessoa em ativida- des sociais, apresentada como grande desafio a ser enfrentado pelos portadores de hipertensão arterial. Embora o índice médio tenha sido de 69,36, maior valor obtido, ele também demonstra comprometimento na qualidade de vida dos pacientes investigados, considerando a qualidade de vida como 100 pontos.

Segundo tem sido descrito, a adaptação social exige energia, criatividade e persistência. Algumas vezes a pessoa com doença crônica limita seu espaço a atividades cotidianas, em decorrência da necessidade de mudar o estilo de vida, bem como o hábito alimentar. Essas situações podem implicar a ausência a reuniões familiares para evitar a ingestão de alimentos inadequados. Assim, a condição crônica de saúde pode levar a diversas perdas nos relacionamentos sociais, nas atividades de lazer e de prazer, conduzindo o paciente ao comprometimento da qualidade de vida.

Na avaliação do domínio "dor", que investiga a presença de sofrimento, fundamentando-se na intensidade da dor, extensão ou interferência nas atividades de vida diária, observaram-se índices muito baixos (56,74 pontos), indicando a interferência deste fenômeno na vida dos entrevistados. A doença hipertensiva é considerada silenciosa e assintomática. Estudos realizados demonstraram que as pessoas, ao serem questionadas sobre dor na hipertensão, referiram sintomas como: dores de cabeça, dores no peito, tontura, alterações nos batimentos cardíacos, alterações visuais e agitações. Conforme se percebe, apesar de a medicina caracterizar os sintomas como inespecíficos, as pessoas apresentam como sintomatologia sensações corpóreas bem específicas, que podem causar interferência nas atividades cotidianas, com prejuízos na qualidade de vida 12,13.

Ao se examinar o domínio "vitalidade", que considera o nível de energia e o de fadiga, obtevese o valor global de 63,32 pontos, sugerindo que conviver com a hipertensão interfere na disposição, ressalvados os comentários relacionados à fadiga ligada à idade, tempo de doença e outros fatores.

O domínio "saúde mental" investiga a presença de ansiedade, depressão, alterações do comportamento, descontrole emocional e bemestar psicológico. Foram apurados 65,45 pontos, indicando que essas situações conduzem os indivíduos ao comprometimento da qualidade de vida.

Ao ser mensurado o domínio "evolução do estado de saúde", conforme se observou, os pacientes percebem seu estado de saúde como muito bom e sua evolução como muito melhor do que há um ano. Como provável explicação para essa manifestação pode-se aventar a idéia de que es- 
tar inscrito em um programa de tratamento, no qual recebe atenção da equipe de saúde e medicamentos, torna a qualidade de vida do portador de hipertensão arterial melhor do que antes. Esse fato pode estar relacionado ao tratamento e à conscientização do paciente acerca da doença, em razão, muito provavelmente, do desempenho dos profissionais que o atendem.

O conjunto de informações obtidas pelos valores entre os diversos domínios indica comprometimento em todos os níveis da qualidade de vida dos portadores de hipertensão arterial analisados entre os diferentes domínios do instrumento SF-36, sugerindo que a hipertensão, provavelmente, conduz homens e mulheres a um comprometimento global de suas vidas ao longo dos anos com a presença da doença. Pela análise estatística, apenas o domínio "estado geral de saúde" mostrou distribuição relevante $(\mathrm{p}<0,05)$.

Na Tabela 2, observa-se a percepção dos pacientes acerca de sua doença e a correlação com os índices de qualidade de vida. Em geral, aqueles com maior prejuízo na qualidade de vida perceberam a doença como grave, embora o outro grupo também tenha apresentado comprometimento na qualidade de vida.

Ao responder ao SF-36, no cômputo dos dados tem-se o seguinte no domínio "estado geral de saúde": estes pacientes perceberam a hipertensão como uma situação grave em suas vidas $(p<0,05)$. Infere-se desses resultados que, por si, a doença prejudica o cotidiano dos portadores de hipertensão arterial. O índice médio encontrado $(51,29)$ foi uma pontuação inferior entre todos os índices.
Observa-se comprometimento da qualidade de vida nos dois grupos de pacientes quando avaliam a percepção de gravidade da doença em todos os domínios, ao ser comparado aos índices globais nas diferentes dimensões, cuja variação foi de 53,58 a 69,36.

Embora não apresente diferença estatística, de acordo com a Tabela 2 o domínio "aspectos emocionais" indica melhor qualidade de vida (75), quando correlacionado com a percepção da hipertensão como uma doença sem gravidade. Tal estado pode estar associado ao enfrentamento e auto-estima preservados entre os pacientes, mesmo em decorrência da doença. Esse fato pode ter conduzido os indivíduos a perceberem a doença como uma situação sem gravidade.

Conforme divulgado por estudo que avaliou as crenças e a percepção da severidade da doença cardiovascular, esta última sofre alterações em decorrência do grau de perturbação emocional criada ao pensar na doença e nas conseqüências que ela pode acarretar, e é influenciada pelas variáveis psicossociais e estruturais do indivíduo 14. Assim, os resultados obtidos na avaliação da percepção da gravidade nosológica sofrem forte influência pessoal, bem como a qualidade de vida sofre repercussões advindas do período diagnóstico da doença, da cultura, dos valores e impressões dos pacientes adquiridos ao longo da vida, fundados em um leque de situações que ultrapassam as informações obtidas pelos profissionais de saúde durante os atendimentos 7,15.

Dessa forma, o fato de o indivíduo ser rotulado como portador de hipertensão pode conduzi-

Tabela 2

Medidas descritivas dos domínios do SF-36 e percepção da gravidade da doença entre os portadores de hipertensão arterial. Fortaleza, Ceará, Brasil, 2003.

\begin{tabular}{|c|c|c|c|c|}
\hline \multirow[t]{4}{*}{ Domínios } & \multicolumn{4}{|c|}{ Percepção dos pacientes sobre a doença } \\
\hline & Sem gravidade & Grave & Total & $p$ * \\
\hline & $(n=20)$ & $(n=93)$ & $(\mathbf{N}=113)$ & \\
\hline & Média \pm DP & Média \pm DP & Média \pm DP & \\
\hline Estado geral de saúde & $64,25 \pm 18,80$ & $51,29 \pm 22,08$ & $53,58 \pm 22,02$ & 0,017 ** \\
\hline Capacidade funcional & $73 \pm 27,31$ & $64,84 \pm 27,93$ & $66,84 \pm 27,88$ & 0,184 \\
\hline Desempenho físico & $57,5 \pm 42,22$ & $60,75 \pm 39,60$ & $60,8 \pm 39,90$ & 0,768 \\
\hline Aspectos emocionais & $75 \pm 38,81$ & $62,37 \pm 40,89$ & $64,60 \pm 40,65$ & 0,183 \\
\hline Aspectos sociais & $67,5 \pm 35,68$ & $69,76 \pm 29,62$ & $69,36 \pm 30,62$ & 0,960 \\
\hline Dor & $65,56 \pm 27,19$ & $54,84 \pm 33,49$ & $56,74 \pm 32,61$ & 0,211 \\
\hline Vitalidade & $64,5 \pm 24,38$ & $63,06 \pm 23,65$ & $63,32 \pm 23,68$ & 0,818 \\
\hline Saúde mental & $66 \pm 19,75$ & $65,33 \pm 22,62$ & $65,45 \pm 22,06$ & 0,853 \\
\hline
\end{tabular}

* Teste Mann-Whitney;

** $p<0,05$. 
lo a manifestações de ansiedade e insegurança, sendo o esclarecimento da doença um ponto inicial na manutenção do controle tensional adequado, reafirmando a necessidade de comunicação efetiva entre os pacientes, profissionais e familiares 16 como medida para conduzir a pessoa a melhorar a percepção dos diferentes aspectos da doença.

Pela Tabela 3, observa-se prejuízo na vida daqueles que consideravam a hipertensão como curável ou não (variação de 50,27 a 80,23). Para os domínios "estado geral de saúde", "capacidade funcional" e "desempenho físico" foi observada distribuição estatística significativa $(\mathrm{p}<0,05)$.

O grupo de pacientes segundo os quais a doença era incurável apresentou concomitantemente comprometimento na qualidade de vida. A distribuição observada no domínio "capacidade funcional" $(\mathrm{p}<0,05)$ denota que a percepção da hipertensão como uma doença incurável esteve associada a um comprometimento da qualidade de vida. Ao mesmo tempo, encontrou-se elevada qualidade de vida $(80,26)$ entre os indivíduos que percebiam a doença como curável. Essa indicação se deu em decorrência de os pacientes apresentarem pouco comprometimento na capacidade de executarem tarefas cotidianas.

$\mathrm{O}$ desempenho físico esteve relacionado às atividades físicas. Essa situação é freqüentemente associada ao bem-estar, à saúde ou à qualidade de vida das pessoas, quando os riscos potenciais da inatividade se materializam, levando à perda precoce da satisfação com a vida. O exercício físico regular torna a pessoa mais calma, traz múltiplos benefícios para o sistema cardio- vascular e auxilia no tratamento dos portadores de hipertensão.

Nesse aspecto, ao se avaliar o "desempenho físico" e a percepção dos pacientes sobre a doença em relação à cura, observou-se que a função física prejudicada conduz os pacientes a um desencorajamento ante a doença $(p<0,05)$. A condição crônica de saúde leva os indivíduos a diversas perdas, entre elas a da capacidade física 17. A presença de restrições físicas nos pacientes avaliados pode, por sua vez, ter contribuído para a percepção de incurabilidade da hipertensão.

Conforme descrito, a percepção da hipertensão como doença incurável conduz o paciente a um sentimento de desânimo, tristeza e depressão, e é um agravante no momento de mudar de hábito ou de estilo de vida exigido pela doença 18 .

O desconhecimento da gravidade da doença e da necessidade de tratamento contínuo induz a várias e importantes considerações, que devem estar presentes no acompanhamento pelos profissionais de saúde. Portadores de hipertensão arterial com longo tempo de tratamento apresentavam dificuldades em identificar o diagnóstico da doença ou mesmo em aceitá-lo, pela falta de compreensão relativa à patologia 19 .

A outra observação a se considerar é a seguinte: a percepção do paciente sobre a não-gravidade da doença, diante de outras tantas dificuldades para a realização do tratamento, provavelmente prejudica sua aceitação. Essa situação sugere a necessidade de mudança no enfoque de educação em saúde e na utilização de uma linguagem comum entre os profissionais e os pacientes. Se

Tabela 3

Medidas descritivas dos domínios do SF-36 e percepção da cura da doença referida entre os portadores de hipertensão arterial. Fortaleza, Ceará, Brasil, 2003.

\begin{tabular}{|c|c|c|c|c|}
\hline \multirow[t]{4}{*}{ Domínios } & \multicolumn{4}{|c|}{ Percepção dos pacientes sobre a doença } \\
\hline & Curável & Não curável & Total & $p$ * \\
\hline & $(n=22)$ & $(n=91)$ & $(N=113)$ & \\
\hline & Média \pm DP & Média \pm DP & Média $\pm \mathrm{DP}$ & \\
\hline Estado geral de saúde & $67,27 \pm 21,31$ & $50,27 \pm 20,99$ & $53,58 \pm 22,02$ & $0,001 * \star$ \\
\hline Capacidade funcional & $80,23 \pm 23,23$ & $62,91 \pm 27,84$ & $66,28 \pm 27,88$ & $0,003 * \star$ \\
\hline Desempenho físico & $77,27 \pm 35,28$ & $56,04 \pm 40,02$ & $60,18 \pm 39,90$ & $0,019 * *$ \\
\hline Aspectos emocionais & $69,70 \pm 41,03$ & $63,37 \pm 40,69$ & $64,60 \pm 40,65$ & 0,459 \\
\hline Aspectos sociais & $72,16 \pm 35,13$ & $68,68 \pm 29,60$ & $69,36 \pm 30,62$ & 0,853 \\
\hline Dor & $65,66 \pm 32,15$ & $54,58 \pm 32,53$ & $56,74 \pm 32,61$ & 0,162 \\
\hline Vitalidade & $69,55 \pm 24,20$ & $61,81 \pm 23,43$ & $63,32 \pm 23,68$ & 0,202 \\
\hline Saúde mental & $71,82 \pm 22,54$ & $63,91 \pm 21,79$ & $65,45 \pm 22,06$ & 0,141 \\
\hline
\end{tabular}

* Teste Mann-Whitney;

${ }^{\star *} p<0,05$. 
os profissionais conhecessem melhor o paciente, seus valores, crenças e estilo de vida, poderiam estabelecer melhores estratégias de educação para saúde, identificando sua percepção em relação ao processo saúde/doença e melhor estimulando o processo de adesão à terapêutica, com melhoria da qualidade de vida. Complementarmente às orientações individuais, os profissionais poderiam desenvolver ações educativas em grupo, pela sua maior efetividade, participação e estímulo aos clientes. Essas ações respondem pela percepção de problemas comuns entre os integrantes, os quais são estimulados ao autocuidado, aumentando a adesão ao tratamento e melhorando sua qualidade de vida 8,14.

\section{Considerações finais}

Conforme verificado ao se investigar a qualidade de vida dos portadores de hipertensão arterial, mediante utilização do SF-36, os pacientes apresentaram comprometimento geral em sua qualidade de vida. Nesse caso, os domínios mais evidentes foram aqueles que investigaram o "estado geral de saúde" e a "dor".

No entanto, ao se avaliar a percepção desses mesmos pacientes sobre seu estado de saúde, este foi manifestado como sendo muito bom e melhor do que há um ano. Desse modo, contrariaram a avaliação feita pela SF-36.

Essa resposta não era esperada, pois, pelo encontrado com a aplicação da SF-36, pela cronicidade da doença e pela pauperização dos pacientes, previa-se uma percepção mais pessimista sobre a doença. Tais resultados provavelmente decorrem do fato de que os pacientes encontram um serviço de saúde que disponibiliza profissionais capacitados, medicamentos específicos, trabalhos desenvolvidos em grupo e acompanhamento domiciliar, refletindo a Estratégia Saúde da Família no contexto social da população com melhoria da sua qualidade de vida. Alie-se a tal comentário a característica conformista do brasileiro em relação às políticas públicas nacionais, especialmente em áreas menos favorecidas como as do estudo 20 .

Como observado, os profissionais que trabalham com o portador de hipertensão arterial no Programa Saúde da Família reúnem condições para acompanhar sua trajetória e evolução. Portanto, podem promover reflexão sobre comportamentos e soluções encontradas e, desta forma, contribuir para aumentar a percepção da doença entre portadores de hipertensão arterial e, ao mesmo tempo, melhorar-lhes a qualidade de vida.

\section{Colaboradores}

\begin{abstract}
D. M. S. Brito participou da coleta de dados, desenvolvimento da pesquisa e redação do artigo. T. L. Araújo revisou o artigo. M. T. G. Galvão participou da organização dos dados da pesquisa e da redação do artigo. T. M. M. Moreira participou da organização do projeto, dos dados coletados e de sua análise, e do desenvolvimento do artigo. M. V. O. Lopes contribuiu na organização dos dados da pesquisa e na redação do artigo.

Objetivou-se investigar a qualidade de vida de portadores de hipertensão arterial e associar estes resultados à percepção dos pacientes sobre a gravidade da doença. Foram avaliados 113 pacientes com hipertensão arterial em seguimento em uma unidade de saúde de Fortaleza, Ceará, Brasil, de maio a agosto de 2002, utilizando-se uma escala de avaliação internacional de qualidade de vida, denominada SF-36. Do total de pa-
\end{abstract} cientes, $77 \%$ eram do sexo feminino, grande proporção com idade superior a 50 anos, vivendo com familiares e exercendo algum tipo de ocupação. Pela avaliação da SF-36, observou-se comprometimento da qualidade de vida nos diferentes domínios, embora os pacientes tenham considerado a doença como sem gravidade e curável. Essas circunstâncias podem interferir no acompanhamento da doença ao longo do tempo, situação que compromete sua qualidade de vida. Conforme se conclui, a avaliação pela SF-36 entre os portadores de hipertensão arterial produziu resultados significativos, indicando prejuízo geral na qualidade de vida. De acordo com o apontado por esses resultados, a hipertensão prejudica as dimensões sociais e físicas dos pacientes, mas tal situação poderá ser alterada com prestação de serviços qualificados.

Questionários; Qualidade de Vida; Hipertensão 


\section{Referências}

1. Lessa I. Adulto brasileiro e as doenças da modernidade: epidemiologia das doenças crônicas nãotransmissíveis. São Paulo: Editora Hucitec; 1998.

2. Organización Mundial de la Salud. Control de la hipertensión. Genebra: Organización Mundial de la Salud; 1996

3. Gaiva MAM. Qualidade de vida e saúde. Rev Enferm UERJ 1998; 6:377-82.

4. Nahas MV. Atividade física, saúde e qualidade de vida: conceitos e sugestões para um estilo de vida ativo Londrina: Midiograf; 2001.

5. Ware JE, Sherbourne CD. The MOS 36-item short form health survey (SF-36). Med Care 1992; 30: 473-83.

6. Ciconelli RM. Tradução para o português e validação do questionário genérico de avaliação de qualidade de vida "Medical Outcomes Study 36item Short-form Health Survey (SF-36)" [Tese de Doutorado]. São Paulo: Universidade Federal de São Paulo; 1997.

7. Reis MG. Glashan RQ. Adultos hipertensos hospitalizados: percepção de gravidade da doença e de qualidade de vida. Rev Latinoam Enferm 2001; 9:51-7.

8. Mendel ES. Adherencia al control de los pacientes hipertensos y factores que la influencian. Ciênc Enferm 1997; 3:49-58.

9. Mion Jr. D, Machado CA, Gomes MAM, Nobre F, Kohlmann Jr. O, Amodeo C, et al. IV diretrizes brasileiras de hipertensão arterial. Arq Bras Cardiol 2004; 83 Suppl 4:1-51.

10. Cade NV. O cotidiano e a adesão ao tratamento da hipertensão arterial. Cogitare Enferm 1997; 2:10-5.

11. Maciel CLC. Emoção, doença e cultura: o caso da hipertensão essencial. In: Romano BW, organizador. A prática da psicologia nos hospitais. São Paulo: Editora Pioneira; 1994. p. 2-38.

12. Lima MT, Bucher JSNF, Lima JWO. A hipertensão arterial sob o olhar de uma população carente: estudo exploratório a partir dos conhecimentos, atitudes e práticas. Cad Saúde Pública 2004; 20: 1079-87.
13. Alves MGM. A voz do hipertenso: representações sociais da hipertensão arterial. Um estudo de caso em Jurujuba, Niterói-RJ [Dissertação de Mestrado]. Rio de Janeiro: Escola Nacional de Saúde Pública, Fundação Oswaldo Cruz; 1997.

14. Dela Colleta MF. O modelo de crença em saúde: uma aplicação a comportamentos de prevenção e controle da doença cardiovascular. Brasília [Tese de Doutorado]. Brasília: Universidade de Brasília; 1995.

15. Reis MG. Hipertensão arterial em adultos hospitalizados: fatores de risco, percepção da doença e qualidade de vida [Dissertação de Mestrado]. São Paulo: Escola Paulista de Medicina; 2000.

16. Moreira TMM, Araújo TL. Sistema interpessoal de Imogene King: as relações entre pacientes com hipertensão não aderentes ao tratamento e profissionais de saúde. Acta Paul Enferm 2002; 15:35-42.

17. Trentini M, Silva DGV, Leimann AH. Mudanças no estilo de vida enfrentadas por pacientes em condições crônicas de saúde. Rev Gaúch Enferm 1990; 11:18-28.

18. Péres DS, Magna JM, Viana LA. Portador de hipertensão arterial: atitudes, crenças, percepções, pensamentos e práticas. Rev Saúde Pública 2003; 37:635-42.

19. Magro MCS, Silva EV, Riccio GMG, Guerra GM. Percepção do hipertenso não-aderente à terapêutica medicamentosa em relação a sua doença. Rev Soc Cardiol Estado de São Paulo 1999; 9:1-10.

20. Brito DMS, Araújo TL. Percepção quanto ao resultado do tratamento e gravidade da doença de portador de hipertensão arterial: estudo em uma unidade básica da saúde de Fortaleza-Ceará-Brasil. Enfermería Integral 2005; 69:40-4.

Recebido em 07/Abr/2006

Versão final reapresentada em 01/Ago/2007

Aprovado em 04/Jan/2008 